\title{
Les exportations françaises de pommes face à la contrainte du traitement au froid en transit : un cas de dépendance de sentier institutionnel
}

\author{
Pasquale LUBELLO • MOISA, Montpellier Supagro, CIRAD, CIHEAM-IAMM, INRA, Univ \\ Montpellier, Montpellier pasquale.lubello@supagro.fr \\ Jean-Marie CODRON • MOISA, CIRAD, CIHEAM-IAMM, INRA, Montpellier Supagro, Univ \\ Montpellier, Montpellier jean-marie.codron@supagro.fr \\ Vincent MATHIEU-HURTIGER • CTIFL, Saint Remy de Provence mathieu-hurtiger@ctifl.fr
}

\section{Résumé :}

Dans cet article, les auteurs illustrent le concept de dépendance de sentier avec le cas d'un dispositif de traitement au froid utilisé pendant le transit maritime dans le commerce international des pommes pour empêcher l'introduction ou la dissémination d'organismes de quarantaine dans les pays importateurs. Ils montrent que ce dispositif est aujourd'hui systématiquement requis et fonctionne comme une norme de facto malgré l'existence de modalités de traitement alternatives potentiellement moins onéreuses et tout aussi efficaces. Conformément au cadre théorique mobilisé, les auteurs indiquent les coûts de négociation qu'un pays exportateur devrait supporter pour faire accepter à ses partenaires commerciaux un dispositif alternatif plus efficient, peuvent s'avérer si élevés qu'il devient préférable de continuer à utiliser le dispositif en usage malgré son coût d'adoption supérieur.

MOTS-CLÉS : pommes, commerce international, traitement au froid, dépendance de sentier, coût de négociation

French apple exports faced with the "in-transit cold treatment" constraint: an institutional « path dependence » case-study

Abstract:

The aim of this paper is to illustrate the path-dependency concept with the case of a cold treatment technique, used in apple international trade during sea transit to prevent introduction or dissemination of quarantine organisms in importing countries. We show that this technique is now systematically required and functions as a "de facto" standard, despite the existence of potentially less costly and equally effective alternative treatment modalities. Drawing on the institutional literature on path-dependency, we show that the negotiation costs that an exporting country would have to bear, in order to have its trading partners accept a more efficient alternative mechanism, can be so high that it becomes preferable to continue using the technique in use despite its higher adoption cost. (JEL: Q17, F14, B52, F55).

KEYWORDS: apples, international trade, cold treatment, path dependence, negotiation cost

\section{Version Postprint}

Paru dans Economie Rurale, $n^{\circ} 370,2019,29-46$.

https://doi.org/10.4000/economierurale.7176 


\section{Introduction}

En 1985, l'American Economic Review publie «Clio and Economics of QWERTY» (David, 1985). Dans cet article, qui marquera la réflexion économique en matière d'innovation technologique, l'auteur explique comment une technologie donnée (la disposition des touches QWERTY) a pu s'imposer dans le monde des machines à écrire puis des claviers d'ordinateur, malgré l'existence prouvée d'alternatives plus ergonomiques (la disposition Dvorak) dont le coût de production serait identique. Pour ce faire, David s'inspira des travaux de W.B. Arthur, économiste nord-irlandais ayant formalisé le concept de «dépendance de sentier ». Selon Arthur (1983), en présence de deux ou plusieurs technologies concurrentes et de rendements croissants d'adoption, ce seraient des variables accidentelles, « les petits accidents de l'histoire », à faire la fortune des uns et la perte des autres. Le passé influencerait donc si lourdement le présent et le futur qu'il rendrait la sortie du sentier emprunté économiquement prohibitif: nous parlons alors d'une situation de « verrouillage » technologique.

Reprises par des auteurs de différents bords (Krugman, 1994 ; Pierson, 2000 ; Pomeranz et Topik, 1999), ces idées n'ont pas échappé à l'attention des économistes institutionnalistes et notamment à celle de D. North (1990), qui s'appliqua à transposer le concept de « dépendance de sentier » et de «verrouillage » au domaine institutionnel. De ce fait, la persistance dans le temps de normes ou d'institutions sous-optimales s'expliquerait par un effet de dépendance de sentier. Dans cette situation, les coûts associés à un changement souhaitable seraient tellement élevés qu'ils empêcheraient le changement de se produire et cela malgré l'existence d'alternatives (normes ou institutions) plus efficientes.

L'objectif de notre article est d'utiliser le concept de la dépendance de sentier pour analyser un dispositif de gestion du risque phytosanitaire appelé «traitement au froid en transit». Ce traitement, largement pratiqué dans les échanges internationaux de produits agricoles frais, consiste à exposer pendant la période de transport maritime et pour une durée minimale, la marchandise contenue dans des containers spécifiquement équipés, à des températures avoisinant les $0^{\circ} \mathrm{C}$, l'objectif étant de garantir l'absence d'organismes nuisibles de quarantaine sensibles au froid et donc de respecter les contraintes phytosanitaires imposées par les pays redoutant de tels organismes nuisibles.

Nous montrons que cette pratique est aujourd'hui systématiquement requise dans les échanges internationaux de pommes, malgré l'existence de modalités de traitement alternatives potentiellement moins onéreuses et tout aussi efficaces. Nous nous posons alors la question de savoir pourquoi ces modalités alternatives n'émergent pas spontanément ou ne diffusent pas plus largement. Nous suggérons que les facteurs techniques et économiques ne sont pas toujours la raison principale et que des raisons de nature institutionnelle peuvent être tout aussi importantes. À cet effet, nous étudions le traitement au froid en transit comme une institution formelle, un dispositif, caractérisé par des normes (un cahier de charges) et des supports matériels dédiés (chambres froides, containers, sondes thermiques et ressources humaines), permettant de certifier l'absence de l'organisme nuisible surveillé. Comme tout dispositif, le traitement au froid en transit présente l'avantage de rendre possibles les échanges - qui ne se réaliseraient pas en son absence, car on se limiterait à interdire les importations - et l'inconvénient d'en limiter l'ampleur de deux façons : par le coût d'adoption 
et le coût de négociation.

Nous montrons dans les sections suivantes, que tout dispositif présente un coût d'adoption que l'on peut qualifier de coût de fonctionnement du dispositif. Ce coût, s'ajoutant aux coûts classiques de production et de commercialisation, réduit mécaniquement les volumes échangeables, relativement à ceux qui auraient pu l'être en l'absence d'une obligation de traitement.

Quant au coût de négociation, il peut être défini comme le coût que chaque pays doit supporter lorsqu'il veut faire accepter à ses partenaires commerciaux une modification du dispositif courant ou l'adoption d'un dispositif alternatif potentiellement plus efficient (au sens de moins coûteux à adopter).

Conformément à la littérature néo-institutionnelle mobilisée, nous expliquons la stabilité du dispositif existant, par le niveau élevé des coûts que devraient supporter les pays exportateurs pour négocier un dispositif alternatif, et ceci malgré des coûts d'adoption inférieurs.

Bien que la pratique du traitement au froid en transit concerne plusieurs fruits et plusieurs pays exportateurs, nous nous focalisons sur la pomme et sur la France pour différentes raisons. Premièrement, la pomme est après la banane, le fruit le plus échangé dans le monde (données Faostat) et surtout le plus exporté vers des pays susceptibles d'imposer de fortes contraintes phytosanitaires. Deuxièmement et plus important encore, la pomme est un fruit facile à conserver au froid (jusqu'à un an dans des chambres froides de type Ultra Low Oxygen). Elle permet ainsi aux pays exportateurs d'imaginer des dispositifs de gestion du risque phytosanitaire plus économes que le traitement au froid en transit. D'où son intérêt pour questionner la stabilité du dispositif existant. Quant à la France, $5^{\mathrm{e}}$ exportateur mondial de pommes (données Faostat), ses exportations ont récemment abandonné les marchés nordeuropéens (de plus en plus saturés) pour investir d'avantage les marchés d'Afrique du nord (Algérie pour l'essentiel), du Moyen-Orient (Arabie Saoudite et Emirats Arabes Unis) et d'Asie (Chine, Inde, Thaïlande). Dans ces derniers, le risque de contamination par Ceratitis Capitata est un problème central et le traitement au froid en transit est systématiquement imposé. Ce qui fait des opérateurs français des acteurs représentatifs de notre problématique.

Dans la première partie de cet article nous développons le cadre théorique dans lequel nous inscrivons notre travail. Dans la deuxième partie, nous traçons l'histoire de l'émergence du dispositif de traitement au froid notamment en transit comme principale technique de gestion du risque phytosanitaire. Nous montrons qu'en l'absence d'une norme internationale dans ce domaine, la norme états-unienne s'est imposée comme référence dans les échanges mondiaux. À cet effet, nous mettons l'accent à la fois sur les variables accidentelles ayant conféré un avantage initial à la norme états-unienne et sur les facteurs ultérieurs ayant renforcé sa position dominante. Dans la troisième partie, nous étudions la pratique alternative proposée par les exportateurs français, ses atouts potentiels et ses contraintes, notamment transactionnelles, qui empêchent sa prolifération au niveau international. Nous concluons en soulignant le rôle décisif des coûts de négociation qui demeurent trop élevés pour faciliter l'adoption d'une norme alternative à la norme états-unienne et ceci malgré un coût d'adoption inférieur.

\section{Cadre Théorique}

\section{1 Éléments généraux}

Depuis quelques décennies, nous assistons, dans le domaine des sciences sociales, à une convergence entre économie, gestion et sociologie autour de deux idées fortes.

La première, de portée générale, pourrait être simplifiée de la manière suivante : les échanges marchands n'émergent pas spontanément, ni se réalisent dans le vide. Autrement dit, le 
marché, au sens classique d'un système impersonnel, décentralisé et autorégulateur d'allocation des ressources, n'existerait pas à proprement parler ou il serait particulièrement coûteux à faire fonctionner. Si les sociologues du marché sont plutôt du premier avis, l'échange marchand étant une relation sociale toujours encastrée (Granovetter, 1985 ; Le Velly 2012), les économistes néo-institutionnalistes partagent l'idée de l'existence de coûts d'utilisation du système de prix, les « coûts de transaction » (Coase, 1937 ; Williamson, 1975, 1985).

La mise en lumière des coûts de transaction n'est pas sans lien avec la deuxième idée forte autour de laquelle la convergence s'opère, et que l'on peut résumer avec North (1990) de la façon suivante: Institutions matter ! Pour ce dernier, l'existence de coûts de transaction ne conditionne pas seulement le choix entre arrangements institutionnels alternatifs (marché, hiérarchie et formes hybrides) mais aussi l'environnement institutionnel (coûts de maintien des institutions et ce qui est important dans notre cas d'étude coûts d'émergence de nouvelles institutions).

Pour reprendre la terminologie de North (North, 1990), un jeu, tel un échange entre deux agents économiques, ne peut être joué que s'il existe des règles (les institutions) communément acceptées par les joueurs (les organisations). Ces règles ainsi que leur crédibilité ont pour rôle fondamental de réduire la complexité du monde dans lequel le jeu est pratiqué, en réduisant à la fois le champ des choix possibles et le degré d'incertitude associé à ce champ (et par conséquent les coûts de transaction que l'incertitude engendre pour les organisations).

Le marché émerge lorsqu'il est «encadré » par et «fondé » sur des règles, formelles ou informelles, légales ou culturelles, qui spécifient les limites du champ d'action ainsi que les modalités d'action des joueurs, en rendant leur comportement plus prévisible, en facilitant le développement d'échanges impersonnels et en rendant profitable la division du travail (North, 2005). Sur ce point, l'économie néo-institutionnelle de North, n'est pas très loin des conclusions de la sociologie économique de Granovetter (1985).

Après avoir initialement adhéré à une vision des Institutions plus néo-classique (North et Thomas, 1973), North rappelle toutefois que rien n'assure que les règles que les sociétés se donnent soient les plus justes (au sens de la justice sociale) ou les plus efficientes possibles (au vu d'un objectif de développement économique) et cela pour au moins trois raisons. Premièrement, les règles sont le produit des interactions sociales et reflètent le plus souvent les rapports de force internes à la société. De ce fait, rien n'assure qu'une société donnée produise et sélectionne les règles lui permettant de maximiser le bien-être social plutôt que les avantages économiques d'un groupe en particulier. Deuxièmement, pour que des règles soient efficientes, il faut qu'elles soient accompagnées par des dispositifs d'enforcement efficaces : en l'absence de tels dispositifs, de bonnes règles peuvent produire un mauvais jeu et des mauvais choix, tout comme le feraient des règles mal choisies. Enfin, rien n'assure que des règles «sous-optimales " soient évincées par de meilleures règles. L'évolution vers des règles plus souhaitables, peut s'avérer trop coûteuse d'un point de vue transactionnel et ne pas se réaliser. Nous parlerons alors d'effet de dépendance de sentier et de situation de verrouillage institutionnel.

Sur ce dernier point, North (1990) enrichit l'analyse néo-institutionnelle en articulant les réflexions d'Arthur (1983) sur les rendements croissants d'adoption avec celles de Coase (1960) sur l'allocation optimale en présence de coûts de transaction positifs. L'existence de rendements croissants d'adoption, c'est à dire la probabilité accrue de préférer la norme ou l'institution la plus diffusée, contribue à faire baisser le coût moyen d'adoption de cette norme en la rendant de moins en moins contestable. Par ailleurs, la présence de coûts de transaction positifs, qui traduisent des marchés incomplets et incertains, réduit davantage les marges de sortie du chemin emprunté. Dans de telles situations, des pratiques dont l'origine est souvent 
liée à des «accidents de l'histoire», et qui ont fini par s'imposer avec la logique des rendements croissants d'adoption, peuvent s'avérer sous-optimales d'un point de vue «allocation générale des ressources » mais rester dominantes du fait de la dépendance de sentier.

\subsection{Dépendance de sentier et marché international}

Dans le cas spécifique du commerce international, notamment des produits agricoles frais et des problèmes phytosanitaires auxquels ce commerce est confronté, nous avançons l'idée que les institutions internationales existantes ne sont pas toujours en mesure de créer des règles optimales (au regard du bien-être social), ni de modifier rapidement les règles existantes lorsque des solutions meilleures se présentent. Cela à cause de l'existence d'importants coûts de transaction liés, selon nous, aux modalités de production des normes propres aux instances internationales, à la faible universalité des normes ainsi produites et à leur faible exécutabilité. Pour ce qui est du premier point, un regard au nombre de votants et aux modalités de vote (par unanimité) retenues par la principale instance internationale du commerce (OMC), suffit à expliquer la longueur des temps de décision. Dans le cas de l'Organisation internationale pour la protection des végétaux (OIPV) par exemple, la durée d'élaboration d'une Norme internationale pour les mesures phytosanitaires (NIMP) (oscille entre 5 et 10 ans. Les Institutions internationales n'en sont pas moins utiles pour autant. Elles permettent grâce à la production de quelques règles communes d'encadrer les négociations bilatérales et d'en réduire les coûts de transaction (Keohane, 1982).

Dans le cas du commerce mondial des produits agricoles frais, la difficulté à produire une règle internationale universelle tient au caractère local des problèmes phytosanitaires détectés. Malgré la vocation universaliste des institutions internationales du commerce ${ }^{1}$, les échanges mondiaux de ce type de produits sont fortement conditionnés par l'existence de risques phytosanitaires à géographie variable, c'est à dire propres aux origines géo-climatiques des couples pays-exportateurs/pays-importateurs et des couples produits/organisme de quarantaine.

L'hétérogénéité des situations dont il est ici question empêche de fait l'émergence de normes homogènes pouvant s'appliquer dans n'importe quel cas de figure (Castonguay, 2005). La preuve en est que les deux principaux accords internationaux en matière de gestion du risque phytosanitaire (Accord sur les mesures sanitaires et phytosanitaires - par la suite «Accord SPS » et Convention internationale pour la protection des végétaux - par la suite «CIPV») délèguent à chaque pays membre le droit de fixer les normes phytosanitaires qu'il estime les plus opportunes ${ }^{2}$, soit de manière unilatérale soit bilatérale. Cette situation limite le rôle des accords internationaux à la production de normes-cadre pour laisser les intérêts particuliers s'exprimer dans les négociations bilatérales.

Enfin, au sujet de la faible exécutabilité des normes internationales deux échelles d'analyse peuvent être empruntées : la première internationaliste, la deuxième bilatéraliste.

Lorsque nous considérons le marché international comme un espace d'échange cadré par des règles communes et gouverné par des instances internationales de contrôle (c'est l'image que nous avons de l'OMC et de l'Organisme de Résolution des Différends par exemple), force est de constater que, historiquement, en l'état actuel, ces instances ne disposent pas de la souveraineté nécessaire pour rendre exécutoires (en dernière instance) à la fois les normes contenues dans les accords internationaux (soient-ils bilatéraux ou multilatéraux), les résolutions éventuellement suggérées par des tribunaux arbitraux internationaux en cas de

\footnotetext{
1 Voir l'article 1 de l'accord de Marrakech (1994), le préambule de l'Accord sur les mesures sanitaires et phytosanitaires (SPS, 1995) ou encore le texte de la norme NIMP 28 (2017).

${ }^{2}$ Art. 2 et 3 de l'accord SPS, 1995.
} 
contentieux, ou encore les contrats privés: d'où l'importance des instances nationales (Berkowitz et al., 2004). La preuve d'un tel manque de pouvoir, en serait la tendance chez les agents commerciaux privés à intégrer dans leurs contrats commerciaux des clauses précisant quelle juridiction nationale appliquer, les modalités concrètes de résolution des différends ou encore la chambre d'arbitrage international à laquelle se soumettre (Casella, 1996), ainsi que la persistance dans le temps de nombreux contentieux commerciaux internationaux irrésolus. Lorsque nous envisageons le commerce international comme une simple somme de relations bilatérales, c'est «l'effet frontière », entendu ici comme fracture entre juridictions nationales (Turrini et Ypersele, 2002), qui serait la cause la plus immédiate de la faible exécutabilité des contrats. Cette idée est proche de celle avancée par nombre d'auteurs ayant travaillé sur la relation entre qualité des institutions (nationales), flux commerciaux et croissance économique (Anderson et Marcouiller, 2002 ; Rodrik et al., 2004).

Les organisations (notamment publiques) qui définissent les règles du jeu sont les états et l'ampleur des échanges entre les pays dépendra essentiellement de la qualité de leurs institutions respectives (Anderson et Mercouiller, 2002) ou bien de leur degré de similarité/dissimilarité (Disdier et Mayer, 2007). Des institutions de mauvaise qualité, notamment celles des pays importateurs (Berkowitz et al., 2004), ou juridiquement trop différentes entre elles, conduiraient toutes à une augmentation du risque de non-exécution des contrats, qui causerait alors une hausse des coûts de transaction, dont les effets seraient les mêmes que ceux d'une taxe à l'importation, et donc à une réduction des flux commerciaux (Lavallée, 2016).

Les éléments présentés ci-dessus suggèrent que les échanges internationaux de produits agricoles frais se réalisent dans un environnement institutionnel lent, fragmenté, et difficilement exécutable, donc incertain. Une telle incertitude pousse à l'émergence de dispositifs normatifs complémentaires ou alternatifs : ces derniers peuvent prendre la forme de normes publiques nationales ayant une plus ou moins grande reconnaissance internationale, ou de standards privés, s'imposant peu à peu comme référence des échanges mondiaux (Lamanthe, 2007). Les uns comme les autres réduisent l'incertitude contractuelle, permettent les échanges impersonnels, en alimentent à long terme la confiance entre les agents économiques. Mais comme toute institution formelle, rien ne nous assure qu'elles émergent du fait de leur efficience à optimiser les coûts de l'échange ou qu'elles persistent pour les mêmes raisons (David, 1985).

À partir de ces quelques éléments de réflexion, nous allons nous concentrer sur trois aspects de la norme dite de traitement au froid. Premièrement, en montrant que, dans l'impossibilité d'une norme internationale commune, la définition de la pratique dite de traitement au froid revient aux nations. Deuxièmement, en montrant que parmi les définitions nationales existantes (et potentiellement concurrentes), celle des États-Unis a réussi à s'imposer comme référence internationale. Elle l'a fait à l'issue de quelques accidents de l'histoire que nous chercherons à caractériser. En montrant enfin que, si aujourd'hui le traitement au froid (selon la définition états-unienne) est quasi systématiquement réalisé en transit pour des raisons de praticité ou de confiance de la part du pays importateur, il l'est aussi à cause de l'absence d'alternatives crédibles. Nous avançons l'hypothèse que l'absence d'alternatives ne s'explique pas principalement par des désavantages économiques ou techniques particuliers, mais par la présence de rendements croissants d'adoption pour le dispositif dominant (partie 2), et par la présence d'importants coûts de transaction dans la sphère des négociations internationales (partie 3$)^{3}$. Ces facteurs, en même temps qu'ils renforcent la position dominante de la norme

\footnotetext{
3 Dans cet article, nous nous intéressons exclusivement aux coûts de transaction de l'environnement institutionnel. Bien que des coûts de transaction au niveau des arrangements institutionnels existent, nous ne les traitons pas ici. Ces coûts sont importants au stade de l'émergence d'une nouvelle norme mais diminuent avec sa diffusion du fait des rendements croissant d'adoption.
} 
de référence, empêcheraient l'émergence de solutions alternatives, préfigurant ainsi une situation dans laquelle le coût de maladaptation (Williamson, 1985) engendré par les règles existantes est inférieur au coût de leur renégociation.

\section{Le traitement au froid en transit : émergence d'une pratique standard}

\subsection{Absence d'un référentiel international pour le couple pomme/MMF}

Signé en 1987 et entré en vigueur en 1989, le protocole de Montréal a pour but de réduire (voire proscrire) la production et l'usage des gaz ayant un impact sur la couche d'ozone. Parmi ces gaz, se trouve le Methyl Bromide. Fortement utilisé en agriculture pour la désinfection des sols, il est aussi largement utilisé pour la désinfection à large spectre des produits agricoles frais destinés à l'exportation ainsi que des palettes en bois, des containers et des cales de bateaux utilisés pour le transport international (MBTOC, 2011).

En 1992, toujours dans le cadre de la convention de Montréal, le Methyl Bromid Technical Options Committee (MBTOC) est chargé d'étudier l'ensemble des alternatives praticables au Méthyl Bromide. En 1994, lors de la publication du premier rapport de la commission (MBTOC, 1995), le traitement au froid est présenté comme un substitut efficace au Méthyl Bromide (aux côtés du traitement par le chaud ou l'irradiation), notamment pour le contrôle des insectes tropicaux, généralement sensibles au froid.

Bien que la MBTOC ait indiscutablement contribué à donner plus de visibilité internationale à la pratique du traitement au froid, il serait erroné de considérer que ces travaux aient abouti à une quelconque définition opérationnelle internationale de la pratique en question. Après plus de 20 ans de travaux de la Commission (MBTOC, 2011) et de nombreux articles académiques en la matière (OEPP, 1990 ; Jessup et al., 1993 ; De Lima, 2007), il n'existe pas de définition précise de ce qu'est le traitement au froid mais seulement des généralités : une pratique consistant à garder les fruits dans un lieu étanche, à une température à cœur de fruit proche de $0 \mathrm{C}^{\mathrm{o}}$ pendant un nombre minimum de jours consécutifs. Aucun protocole universel n'a émergé, et aucune norme internationale permettant de guider les acteurs n'a été promue par des instances internationales ad hoc comme l'OIPV ${ }^{4}$.

Conformément aux éléments théoriques rappelés plus haut, cette absence de norme universelle serait la conséquence naturelle de la forte hétérogénéité des situations phytosanitaires dans lesquelles l'échange international de produits agricoles frais se réalise. Cette même hétérogénéité matérielle conduit à ce que les restrictions phytosanitaires (leur définition précise) soient élaborées à une échelle institutionnelle moindre, nationale, et donc forcément plurielle. Qu'il n'existe donc pas de norme internationale mais plusieurs déclinaisons nationales de la pratique du traitement au froid, est tout à fait normal. Nous avançons en revanche l'idée qu'en l'absence d'une norme internationale «de jure », c'est à dire élaborée au sein même des organisations internationales, et face à la forte variabilité des normes élaborées à l'échelle nationale, une norme nationale particulière (la norme USDAAPHIS) se soit imposée comme référence internationale de facto, c'est à dire, de par sa capacité à faire consensus ou à s'imposer parmi les utilisateurs (les entreprises).

\section{2 Émergence du référentiel USDA/APHIS : accidents de l'histoire et rendements croissants d'adoption}

Face à l'absence d'une norme internationale en matière de traitement au froid des pommes pour le contrôle de la mouche méditerranéenne, un examen attentif des contraintes phytosanitaires imposées par les pays importateurs à un panel représentatif de pays

\footnotetext{
${ }^{4}$ Exception faite pour le contrôle de la mouche méditerranéenne sur les citrus (NIMP 28). Source : OIPV.
} 
exportateurs de pommes, nous conduit à qualifier la norme USDA-APHIS (et notamment le protocole T107-a ${ }^{5}$ ) de référentiel dominant (tableau 1$)^{6}$.

Dans le cas de la France par exemple, sur douze destinations imposant le traitement au froid pour le contrôle de Ceratitis Capitata, cinq requièrent le respect de la norme USDA-APHIS T107. Pour l'Afrique du Sud, sur douze destinations répertoriées, quatre imposent la norme USDA-APHIS. La même proportion (un tiers) dans le cas australien. Quant à l'Italie, compte tenu du peu de données disponibles sur le couple pomme/mouche méditerranéenne (un seul protocole bilatéral signé, notamment avec les USA), nous nous sommes concentrés sur un produit proche : le kiwi. Le résultat ne change guère : la norme USDA-APHIS T107 parait toujours dominante. D'autant plus si on se limite aux seuls pays asiatiques.

Tableau 1. Normes de traitement au froid pour le couple Ceratitis Capitata / pomme et pour les principaux pays importateurs (hors USA)

\begin{tabular}{|c|c|c|c|}
\hline $\begin{array}{l}\text { FRANCE } \\
\text { vS }\end{array}$ & NORME & $\begin{array}{l}\text { AFRIQUE DU } \\
\text { SUD vS }\end{array}$ & NORME \\
\hline Algérie & $1 \% / 15$ jours & Bangladesh & $0 \% / 14$ jours \\
\hline Bangladesh & $0^{\circ} / 14$ jours & Chine & $1 \% / 16$ jours \\
\hline Chine & $1 \% / 14$ jours & Ghana & Norme USDA-APHIS T107-a \\
\hline Inde & Norme USDA-APHIS T107-a & Inde & Norme USDA-APHIS T107-a \\
\hline Iran & Norme USDA-APHIS T108-a & Indonésie & Norme NIMP 28* \\
\hline Jordanie & $1 \% / 40$ jours & Jordanie & $1,5 \% 14$ Jours \\
\hline Maurice & Norme USDA-APHIS T107-d & Madagascar & $0 \% 15$ jours \\
\hline Nigeria & $\begin{array}{l}0^{\circ} / 5 \text { jours ou } 2^{\circ} / 10 \text { jours ou } 3 \% 14 \\
\text { jours }\end{array}$ & Maurice & Norme USDA-APHIS T107-d \\
\hline Sri-Lanka & $\begin{array}{l}0^{\circ} / 14 \text { jours ou } 0,55^{\circ} / 18 \text { jours ou } \\
1,1^{\circ} / 20 \text { jours ou } 2,2^{\circ} / 22 \text { jours }\end{array}$ & Nigeria & $\begin{array}{l}0^{\circ} / 5 \text { jours ou } 2^{\circ} / 10 \text { jours ou } \\
3^{\circ} / 14 \text { jours }\end{array}$ \\
\hline Taiwan & $\begin{array}{l}0^{\circ} / 12 \text { jours ou } 1,7^{\circ} / 14 \text { jours ou } \\
3,3^{\circ} / 18 \text { jours }\end{array}$ & Sri-Lanka & $\begin{array}{l}0^{\circ} / 14 \text { jours ou } 0,55^{\circ} / 18 \text { jours } \\
\text { ou } 1,1^{\circ} / 20 \text { jours ou } 2,2^{\circ} / 22 \\
\text { jours }\end{array}$ \\
\hline Thaïlande & Norme USDA-APHIS T107-a & Taiwan & $\begin{array}{l}0^{\circ} / 12 \text { jours ou } 1,7^{\circ} / 14 \text { jours ou } \\
3,3^{\circ} / 18 \text { jours }\end{array}$ \\
\hline Vietnam & Norme USDA-APHIS T107-a & Zambie & Norme USDA-APHIS T107-a \\
\hline ITALIE vS & & AUSTRALIE vs & \\
\hline Chine & Norme USDA-APHIS T107-a & Îles Solomon & $0 \% 13$ jours ou $1 \% 16$ jours \\
\hline Inde & Norme USDA-APHIS T107-a & Indonésie & Norme NIMP 28 \\
\hline Japon & Norme USDA-APHIS T107-a & Jordanie & $1 \% / 40$ jours \\
\hline $\mathrm{NZ}$ & Norme USDA-APHIS T107-a & Philippines & Norme USDA-APHIS T107-a \\
\hline \multirow[t]{2}{*}{ Taiwan } & $\begin{array}{l}0^{\circ} / 12 \text { jours ou } 1,7^{\circ} / 14 \text { jours ou } \\
3,3^{\circ} / 18 \text { jours }\end{array}$ & Polynésie Fr & $0^{\circ} / 10$ jours ou $2,5^{\circ} / 16$ jours \\
\hline & & Thaillande & Norme USDA-APHIS T107-a \\
\hline
\end{tabular}

Note : *Norme NIMP 28 Ceratitis (citrus) $\left(2^{\circ} / 16\right.$ jours ou $3^{\circ} / 20$ jours).

Conformément au cadre théorique mobilisé plus haut, la position dominante acquise par le référentiel USDA/APHIS doit être expliquée par des accidents de l'histoire lui ayant conféré un avantage initial, et par la présence d'éléments factuels signalant l'existence de rendements

\footnotetext{
${ }^{5}$ Cette norme a pour qualité de spécifier non seulement les couples température/jours visant à traiter par le froid la mouche méditerranéenne pour un large spectre de fruits, mais aussi tous les détails concernant le nombre et les modalités d'emplacement des sondes enregistreuses (aériennes ou à cœur de fruit) dans les celles frigorifiques ou dans les containers : https://www.aphis.usda.gov/aphis/ourfocus/planthealth/complete-list-of-electronic-manuals.

6 Source France: https://teleprocedures.franceagrimer.fr/Expadon/Login/Login.aspx?ReturnUrl=\%2fexpadon. Source Italie : https://www.politicheagricole.it/. Source Afrique du Sud : https://ppecb.com/documents/. Source Australie : http://www.agriculture.gov.au/export/micor.
} 
croissants d'adoption.

Bien avant les inquiétudes du milieu des années 1990 concernant la dégradation de la couche d'ozone, d'autres accidents de l'histoire ont contribué à faire émerger cette pratique. L'apparition de Ceratitis Capitata à Perth (Australie) en 1895 (Bonizzoni et al., 2004), puis l'infestation, en 1907, des îles Hawaii du fait de ses relations commerciales avec l'Australie (Vargas et alii, 2001), ainsi que les crises enregistrées en Floride en 1929, au Texas en 1966 et pour finir en Californie dès $1975^{7}$, ont conduit les nord-américains à expérimenter, depuis la fin du $19^{\mathrm{e}}$ siècle, l'usage du froid comme mesure phytosanitaire préventive contre la mouche méditerranéenne, notamment dans le cadre du commerce domestique de fruits frais (Wilcox et Hunn, 1914).

Si les crises de contamination rappelées à l'instant, permettent d'abonder dans le sens d'un avantage temporel des nord-américains dans la lutte contre Ceratitis Capitata et notamment en matière de traitement au froid, elles ne suffisent pas pour autant à expliquer la diffusion dans l'espace, de la norme américaine. Pour cela, il faut rappeler quelle était la position des ÉtatsUnis dans le commerce mondial des pommes au cours du $20^{\mathrm{e}}$ siècle. Contrairement à l'Europe (France et Italie notamment), les États-Unis ont toujours eu pour particularité d'orienter leurs exportations vers des pays à problématiques phytosanitaires fortes (les pays asiatiques notamment). Avec ces derniers, des protocoles bilatéraux ont été signés bien avant l'arrivée des européens (depuis les années 1950 pour les États-Unis, courant des années 2000 pour la France).

Si la précocité avec laquelle les États-Unis ont été confrontés au risque de contamination par Ceratitis Capitata, à la fois en tant qu'importateurs et exportateurs, participe à l'explication de l'avantage initial acquis par la norme USDA/APHIS, d'autres facteurs plus récents s'ajoutent, dans une dynamique de renforcement de la norme par feedback positifs.

Depuis les années 2000, l'USDA-APHIS procède à l'agrément de tous les bateaux, containers et sondes d'enregistrement utilisés pour importer des produits frais sur le territoire américain (base de données $\mathrm{CPHST}^{8}$ ). L'autorité phytosanitaire s'assure ainsi que le traitement au froid des produits frais importés est conforme aux conditions décrites par le référentiel national.

Bien que l'agrément en question soit un instrument de contrôle des seuls flux entrants (importations), nous avançons l'idée que ces bateaux, containers ou sondes agrées fonctionnent tels des vecteurs de diffusion de la norme états-unienne dans le monde. En ce sens et conformément aux éléments rappelés dans le cadre théorique, s'il est vrai qu'une entreprise de fret maritime a d'autant plus d'intérêt à se conformer à la norme états-unienne, que le nombre de pays importateurs adoptant la même norme augmente, il est aussi vrai que l'augmentation du nombre de sociétés de fret maritime agréées par l'USDA-APHIS, accroît parallèlement la probabilité qu'un pays importateur adopte le standard américain. Cette probabilité est accrue par défaut, c'est à dire par le simple fait qu'il devient statistiquement plus probable de travailler avec une compagnie agréée qu'avec une non agréée, ou du fait d'une recherche de simplification de la part de l'exportateur, qui préfère répondre à une norme phytosanitaire universelle plutôt qu'à une multitude de règles bilatérales.

À ce titre, rappelons que dans des protocoles bilatéraux d'échange signés par l'Italie, l'Afrique du Sud ou l'Australie, avec notamment des pays asiatiques (Chine, Thaïlande, Inde, Indonésie ou Iran entre autres), il est explicité que le traitement au froid doit être réalisé en transit et exclusivement sur des bateaux-cale ou dans des containers agrées par l'USDAAPHIS, ou encore avec des sondes certifiées par l'USDA-APHIS. Toujours à ce sujet, rappelons aussi que des compagnies maritimes comme APL ou CMA-CGM, effectuent, depuis plusieurs années, le traitement au froid en transit exclusivement avec des équipements agréés par l'USDA-APHIS et cela quelle que soit la destination finale.

\footnotetext{
${ }^{7}$ URL : http://entnemdept.ufl.edu/creatures/fruit/mediterranean_fruit fly.htm\#top

${ }^{8} \mathrm{URL}: \underline{\text { https://vessels.cphst.org/index.cfm }}$
} 
Enfin, rappelons que tout pays exportateur de pommes souhaitant exporter vers les États-Unis doit accepter que des inspecteurs phytosanitaires états-uniens forment les inspecteurs sanitaires des pays partenaires aux techniques de gestion du risque phytosanitaire propres à la norme USDA-APHIS. La procédure de traitement au froid, notamment en transit, est au cœur de cette formation. Si l'on considère que les frais de formation (le coût d'apprentissage) sont à la charge du pays exportateur, nous pouvons alors ici aussi supposer que le pays exportateur s'engageant dans une relation commerciale coûteuse, privilégiera parmi ses autres partenaires commerciaux, ceux qui adoptent la même norme, dans le but de rentabiliser son investissement initial.

Les éléments ici évoqués, nous conduisent à qualifier la norme USDA-APHIS, de référentiel international. Un référentiel qui, comme nous venons de le voir, ne se limite pas à la seule dimension normative, mais y associe des dispositifs à la fois matériels (moyens certifiés) et humains (personnel agrée, formations) qui la renforcent.

\section{3) Le « traitement au froid en transit : une pratique institutionnellement dépendante}

\subsection{Les avantages techniques et économiques du traitement en transit}

Selon les informations recueillies auprès des opérateurs de la filière pomme française (ANPP, Interfel, Aneefel, Coopératives ${ }^{9}$ ), la quasi-totalité des pommes exportées vers des pays imposant le traitement au froid, reçoit ce traitement en transit, c'est à dire pendant le transport, dans des containers réfrigérés équipés de sondes enregistreuses. Toujours à partir des informations mises à disposition par ces opérateurs, le coût de cette opération est certes variable (selon la compagnie maritime employée) mais d'environ $1100 € /$ container.

Face à ce coût, qui correspond au coût d'adoption d'un mode précis de gestion du risque SPS (la norme USDA/APHIS), il est opportun de se demander pourquoi des modalités techniques alternatives de gestion du même risque, qui pourtant existent, n'émergent pas, du moins en France. La première réponse à la question passe par la mise en avant des avantages techniques, économiques et institutionnelles de la pratique courante (le traitement au froid en transit).

Son avantage le plus frappant réside sans doute dans le fait de réaliser deux opérations (transport et traitement au froid) en même temps. Le gain de temps (variable selon la destination) qui en découle, cache deux autres avantages : premièrement, le fait de réaliser le traitement en pleine mer contribue à limiter toute forme de contamination ex-post et constitue un avantage technique ; deuxièmement, le fait de pouvoir travailler à flux tendu constitue un avantage économique non négligeable car il permet de réagir au mieux au prix de marché, notamment en début de campagne. Toute pomme convenablement conditionnée et prérefroidie peut partir, alors qu'un traitement au froid à l'origine retarderait de plusieurs semaines le départ du bateau, affectant potentiellement les capacités de négociation des exportateurs. Un autre avantage technique non négligeable du traitement au froid en transit est son faible taux d'échec apparent ${ }^{10}$. Dans le cas français par exemple, le seul cas recensé concerne les exportations vers la Thaïlande: pendant l'année d'ouverture du marché thaïlandais (en 2012-2013) un container sur trois a été rejeté à cause d'un traitement défaillant dû à un mauvais positionnement des sondes et à des variations trop importantes de la

\footnotetext{
${ }^{9}$ Informations recueillies dans le cadre du projet ANR Sustain'Apple (2014-2018).

${ }^{10}$ Les échecs ne font pas toujours l'objet d'un contentieux ou d'une communication auprès des instances publiques. Ils peuvent être résolus en interne par les opérateurs privés (exportateurs et importateurs) par le biais de réexportations vers des destinations moins exigeantes.
} 
température enregistrée ${ }^{11}$.

Aux avantages techniques et économiques, s'en ajoute un dernier que nous qualifions de transactionnel. Nous pouvons raisonnablement imaginer que le fait de déléguer la responsabilité de la bonne réussite du traitement au froid au transporteur maritime, un opérateur privé, puisse être perçu par les opérateurs commerciaux (exportateurs et importateurs) comme un gage de sécurité contractuelle quant aux opérations effectuées. Dans ces cas, c'est la renommée de l'opérateur privé qui est en jeu et non plus la crédibilité d'agences phytosanitaires nationales et souveraines. L'émergence récente de systèmes d'assurance ou de contrôle à distance en matière de traitement au froid, proposés par les principales compagnies de fret maritime ${ }^{12}$, alimente le sentiment de confiance quant à la fiabilité des opérations ainsi déléguées.

Dans les deux sections suivantes nous démontrons que, des trois dimensions ci-dessus rappelées, la plus contraignante est in fine la dimension transactionnelle notamment lorsque nous nous plaçons à l'échelle des négociations visant à modifier le dispositif adopté (donc de l'environnement institutionnel).

\subsection{Les avantages techniques et économiques du traitement en chambre froide}

Le seul substitut connu du traitement au froid en transit est le traitement au froid en chambre froide (MBTOC, 2011). Ce dernier peut être réalisé à l'origine, dans les chambres froides des stations fruitières des pays exportateurs, ou à l'arrivée, dans les chambres froides portuaires des pays importateurs. Rappelons que les premières ont une fonction classique de conservation des fruits, tandis que les deuxièmes ont le plus souvent comme fonction celle de réaliser des traitements d'émergence en cas de défaillance des traitements en amont ${ }^{13}$.

Des deux options, la deuxième semble la moins praticable, pour plusieurs raisons : les ports ne sont souvent pas suffisamment équipés de chambres froides spécifiques pour le traitement au froid, le coût est souvent plus élevé lorsque des traitements d'urgence sont réalisés à l'étranger et enfin les opérations techniques ainsi que la qualité finale du produit échappent au contrôle des exportateurs. C'est pourquoi nous nous concentrons pour la suite, sur le traitement au froid à l'origine.

D'un point de vue technique, les chambres froides présentent les mêmes avantages voire plus d'avantages qu'un container. Tout d'abord, dans le cadre des stations de stockage et conditionnement certifiées insect proof, le risque de contamination ex-post est réduit et guère supérieur à celui d'un container en pleine mer.

Ensuite, tandis qu'une chambre froide est conçue pour générer de grandes quantités de froid, un container réfrigéré ne peut que maintenir une température donnée et corriger d'éventuelles petites fluctuations de température (Foulquier, 2007). De ce fait, le container réfrigéré ne peut être opérationnel qu'après un refroidissement en chambre froide (ce que l'on appelle precooling), tandis que la chambre froide peut assurer seule l'ensemble des opérations. Dans l'hypothèse dans un traitement au froid à l'origine, la mouche étant déjà éradiquée, un

\footnotetext{
${ }^{11}$ Source : entretien avec Pierre Varlet (ANPP).

${ }^{12}$ C'est le cas de la Garantie Cold Treatment proposée par la compagnie CMA-CGM, la garantie Cold Treatment Assured proposée pat la compagnie APL, le service de Cold Treatment proposé par COSCO, ou encore le service de suivi à distance des containers réfrigérés (RCM) proposé par MAERSK, pour ne citer que quelques exemples. Faisons observer en passant que les investissements consentis par ces entreprises afin de garantir davantage le bon déroulement des opérations en transit, renforcent la fiabilité de ce dispositif particulier de gestion du risque de contamination par Ceratitis Capitata.

${ }^{13}$ Elles peuvent avoir aussi une fonction de stockage, lorsque les calendriers fiscaux changent en cours de campagne. Dans les pays où les tarifs douaniers changent en cours d'année (c'est le cas actuellement de l'Inde, par exemple, autrefois le cas de l'UE), l'incitation est forte pour les exportateurs de profiter de la période fiscale la plus avantageuse pour faire rentrer le plus possible de marchandises. Dans cette situation, il est prévisible que les entrepôts frigorifiques au niveau portuaire soient largement sollicités pour du stockage à froid.
} 
container classique serait suffisant pour effectuer le transport maritime.

Quant aux avantages économiques d'un traitement au froid à l'origine, ils sont aussi nombreux. Premièrement, le traitement au froid à l'origine, étant centralisé et contrôlable en continu, permet à l'exportateur de réagir rapidement en cas d'échec de la procédure. En ce sens, l'échec du traitement au froid, bien qu'il implique une perte de temps (nécessité de recommencer le traitement), n'implique pas à priori la destruction de la marchandise ou son détournement vers un marché moins rémunérateur. Deuxièmement, la plupart des chambres froides françaises fonctionnent habituellement à des températures compatibles avec celles des traitements requis par les pays importateurs (entre 0,5 et $2,5^{\circ} \mathrm{C}$ ), ce qui n'entraîne aucun coût supplémentaire de fonctionnement. Enfin, les chambres froides permettent de traiter un plus grand volume de pommes que dans un container, procurant ainsi des économies d'échelle et d'organisation.

\subsection{Coûts de transaction dans l'environnement institutionnel}

S'il est raisonnable de penser que l'usage des chambres froides à des fins de traitement à l'origine est plus simple et potentiellement plus économe, la pratique du commerce international nous dit le contraire : la totalité des pommes françaises exportées vers des pays demandant un traitement au froid, le sont en transit. Les blocages à l'adoption d'une pratique alternative se situent en effet au niveau de l'environnement ${ }^{14}$ institutionnel et peuvent être analysés sur deux exemples : la production de preuves scientifiques permettant de débloquer la négociation et la négociation multilatérale.

\section{La voie étroite de la preuve scientifique}

Une démarche de preuve scientifique consisterait à démontrer que, sous certaines conditions, la conservation au froid dans une chambre froide conventionnelle permet d'atteindre les mêmes résultats qu'un traitement au froid tel que normé par l'USDA-APHIS. Il s'agirait alors de «produire des preuves scientifiques suffisantes ${ }^{15}$ qui légitimeraient, dans l'ordre, la demande et l'obtention d'une équivalence technique, puis la renégociation bilatérale des protocoles d'échange. Les expériences de laboratoire menés en France, par le CTIFL (Vincent Mathieu-Hurtiger, forthcoming), vont dans ce sens. Elles ont servi à tester l'hypothèse selon laquelle des pommes, préalablement refroidies (precooling), puis gardées à une température égale ou inférieure à $2.22^{\circ}$ dans une chambre froide conventionnelle pour une durée de 18 jours au moins, ne présentent pas de traces de Ceratitis Capitata (et ce quel que soit le stade de développement de l'organisme de quarantaine).

Malgré les résultats encourageants de l'étude, l'analyse des différends passés en matière de tests expérimentaux sur le traitement au froid, laisse entrevoir des limites majeures à la «voie de la preuve scientifique $»$. Une première limite a trait à l'originalité des preuves scientifiques. En fait, conformément à l'accord SPS (1995), dans le cadre d'une relation bilatérale, chaque pays importateur a le droit d'exiger du pays exportateur qu'il fournisse des preuves scientifiques sur mesure. En ce sens, le pays exportateur ne peut ni emprunter la littérature scientifique produite par d'autres pays ayant déjà effectué des tests expérimentaux sur le même produit et pour le même pays importateur, ni utiliser les preuves scientifiques qu'il aurait lui-même produit dans le cadre d'une négociation bilatérale analogue avec un

\footnotetext{
${ }^{14}$ Comme nous l'avons écrit précédemment, notre article ne s'intéresse pas aux coûts de transaction engendrés par la norme au niveau des arrangements institutionnels. Ceux-ci peuvent être élevés lorsque la norme est émergente mais finissent par disparaître lorsque la norme diffuse largement.

${ }^{15}$ Conformément à l'accord SPS (Art 2, alinéa 2).
} 
autre pays importateur ${ }^{16}$. La raison invoquée serait l'hétérogénéité des conditions géoclimatiques, physiques et/ou variétales dans lesquelles les tests sont réalisés. La preuve scientifique devient alors, au sens de la théorie, un actif spécifique de la négociation bilatérale, ne pouvant être redéployé au cours de la négociation suivante.

La seconde limite a trait à la spécificité des protocoles expérimentaux. De manière similaire à ce que nous venons de voir, chaque pays importateur a le droit d'imposer au pays exportateur de produire une norme scientifique sur mesure à partir d'un protocole expérimental ad hoc. Pour éclairer ce point, rappelons que le Japon, ainsi que la Nouvelle Zélande ou l'Australie, imposent aux pays exportateurs que les tests sur les organismes quarantenaires portent sur un nombre minimum de spécimens (30 milles pour la mouche méditerranéenne) et aboutissent à des seuils de probité de 9 (Follett et Hennessey, 2007), tandis que d'autres pays peuvent avancer des conditions moins strictes. Cela renforce l'idée selon laquelle la méthode de production de la preuve scientifique peut être considérée comme un actif spécifique, difficilement redéployable et générateur de coûts de transaction entre pays et donc au niveau des environnements institutionnels concernés.

\section{Les limites de la négociation multilatérale}

Comme nous l'avons vu dans les sections précédentes, malgré l'avantage technique et économique que l'on a pu associer à l'usage de la conservation au froid en tant que technique alternative de gestion du risque de contamination par Ceratitis, et malgré l'émergence d'études scientifiques menées pour démontrer l'efficacité phytosanitaire d'une telle alternative, la production de la preuve scientifique dans le cadre d'une négociation bilatérale supporte des coûts de transaction élevés, ce qui contribue à expliquer la difficulté d'émergence d'une alternative. Face à ce constat, une autre voie semble envisageable: la négociation multilatérale d'équivalences techniques. Cette procédure consiste à statuer sur une équivalence technique universelle s'imposant quelles que soient les parties en présence dans la négociation bilatérale. Elle réduit donc les coûts de transaction des négociations bilatérales qui peuvent désormais s'appuyer sur la reconnaissance d'un élément technique pour lequel il n'est plus nécessaire d'apporter bilatéralement des preuves scientifiques.

Malgré l'existence de cette voie, nous constatons toutefois qu'une telle procédure n'est pas d'actualité. L'explication réside encore une fois dans la nature même des structures institutionnelles qui gouvernent le marché international des produits agricoles frais et des coûts de transactions qui lui sont associés.

L'iter institutionnel qui doit être parcouru pour qu'une technique de gestion du risque soit reconnue comme équivalente à une technique déjà existante est potentiellement long. Il passe par les commissions d'équivalence technique et/ou d'harmonisation d'instances internationalement reconnues: dans le cas des produits végétaux ce sont l'OIPV, et notamment les groupes de travail intitulés Technical Panel on Phytosanitary Treatment (TPPT), qui discutent des éléments techno/scientifiques apportés par les parties intéressées. Mises à part la longueur de la procédure (5 à 10 ans) et la nature purement consultative du travail de ces panels, une éventuelle reconnaissance internationale d'une équivalence technique est également limitée par le caractère non contraignant d'une telle équivalence, que ce soit pour les négociations déjà effectuées comme pour celles à venir.

\footnotetext{
${ }^{16}$ Rappelons à ce titre, le cas de la négociation entre Chili et Japon en matière de traitement au froid sur la mouche méditerranéenne. Dans les années 1980, le Japon a exigé de participer (présence physique au Chili) à la définition du protocole expérimental et aux expérimentations, visant à montrer l'efficacité du traitement au froid dans la gestion de la mouche méditerranéenne. A la différence d'autres pays asiatiques, (Chine, Taiwan, Thaillande) le Japon ne s'était pas satisfait des preuves scientifiques initialement avancées par le Chili, bien que ces mêmes preuves eussent été validées dans le cadre de négociations similaires avec les USA (informations recueillies en 2016 par J.M. Codron lors d'un entretient avec Roberto Mir du Servicio Agricola y Ganadero, SAG-Chili).
} 
Prenons un exemple récent : en 2016 le TPPT conclut à «l'absence d'évidences scientifiques supportant l'idée selon laquelle des populations de Ceratitis Capitata, originaires de régions géographiques éloignées, réagiraient différemment au froid $\gg^{17}$. Le TPPT qualifie cette conclusion d'avancée majeure dans le processus d'harmonisation des techniques de traitement au froid. Elle permettrait en effet à un pays exportateur de faire valoir les preuves scientifiques déjà apportées par d'autres pays ou par des recherches scientifiques de renommée internationale, en lieu et place de les produire ad hoc. Malgré cela, aucune NIMP relative à ce thème n'a été éditée pour l'instant par l'OIPV et aucun pays ne semble avoir utilisé cet argument dans les négociations les plus récentes.

Les limites de la négociation multilatérale semblent donc confiner les pays au jeu des négociations bilatérales et à leurs contraintes spécifiques: une longue durée de négociation (10 ans en moyenne ${ }^{18}$ ); une négociation qui doit être réitérée avec chaque pays et pour chaque produit, et qui mobilise plusieurs acteurs : les opérateurs du commerce (qui pressent pour l'ouverture de nouveaux pays), les réseaux diplomatiques (qui représentent les intérêts politiques et commerciaux d'un pays), les réseaux d'experts (qui participent à l'analyse de risque du pays) ainsi que les représentants ministériels qui procèdent à la ratification des accords et des protocoles. Le coût de la transaction institutionnelle de mise en place et de fonctionnement d'un tel dispositif est ainsi loin d'être négligeable.

\section{Conclusion}

Les exportations de pommes, originaires de pays hébergeant la mouche méditerranéenne et destinées à des pays exempts de cet organisme quarantenaire, font l'objet d'exigences phytosanitaires particulières, émises par les pays importateurs soit de manière unilatérale soit dans le cadre d'accords phytosanitaires négociés de façon bilatérale (protocoles bilatéraux d'échange). Parmi ces exigences et notamment dans la catégorie des traitements ex post, le traitement au froid semble dominer, notamment de par sa qualité de traitement physique (par opposition aux traitements chimiques ou radioactifs).

Dans le cas des exportations françaises de pommes, notamment à destination de pays sensibles à la mouche, le traitement au froid est systématiquement réalisé « en transit », c'est à dire pendant la période de transport maritime, ce qui engendre un surcoût d'environ 1100 Euros/container comparé à une expédition sans traitement, soit environ $7 \%$ du prix de la pomme.

Dans ce travail, nous avons essayé de comprendre les raisons du succès de cette modalité précise de gestion du risque phytosanitaire, compte tenu de son coût et de l'existence d'autres modalités d'application du traitement au froid, notamment l'usage du froid dans les chambres de stockage des stations de conditionnement françaises.

Nous avons montré que les avantages techniques et économiques que nous pouvons raisonnablement associer à la pratique du traitement au froid en transit peuvent être également obtenus de façon aussi intéressante par la pratique alternative de conservation au froid en chambre froide et ne sauraient donc justifier à eux seuls le traitement en transit pratiqué aujourd'hui de façon quasi exclusive dans le commerce international des pommes. Pour expliquer le non développement des pratiques alternatives, pourtant tout aussi intéressantes sur le plan économique que les pratiques de traitement au froid en transit, il faut mobiliser d'autres types d'arguments, cette fois-ci de nature institutionnelle.

En faisant appel au cadre théorique de l'économie néo-institutionnelle, nous pouvons parler dans ce cas spécifique, de dépendance de sentier. Comme nous l'avons vu dans la section 2, la

\footnotetext{
17 Traduction des auteurs.

${ }^{18}$ Durée confirmée par les opérateurs de la filière pomme française (France Agrimer) et italienne (Assomela).
} 
pratique du traitement au froid en transit émerge aux États-Unis au début du $\mathrm{XX}^{\mathrm{e}}$ siècle dans le cadre du commerce domestique (Hawaii vs reste des Etats fédéraux) de produits agricoles frais, en réponse à des crises phytosanitaires majeures. Toujours dans la section 2, nous avons montré que l'adoption de facto de la norme USDA-Aphis 107 par bon nombre de pays partenaires, notamment asiatiques, ne se limite pas à l'adoption par les opérateurs du commerce d'un simple tableau de correspondance températures/jours, mais aussi de dispositifs matériels et humains agréés par les États-Unis qui l'accompagnent et le renforcent. Dans ce contexte, toute tentative de changement de la norme dominante se heurte à de fortes limites institutionnelles génératrices d'importants coûts de transaction.

À ce titre, nous nous sommes concentrés sur deux freins majeurs de l'environnement institutionnel international en matière d'échanges de produits frais. D'une part, le besoin de production de savoirs scientifiques sur mesure, dont la conformité et la qualité est toujours soumise à l'appréciation du pays importateur. D'autre part, l'impossibilité de négociations multilatérales dans un domaine où l'hétérogénéité des situations géo-climatiques conduit fatalement à des négociations bilatérales. Ces deux contraintes sont productrices de coûts, que nous qualifions pleinement de coûts de transaction propres à l'environnement institutionnel. À ces coûts, il faut rajouter les coûts de transaction engendrés au niveau des arrangements institutionnels au stade d'émergence de la norme. C'est finalement l'ensemble de ces coûts de transaction, plutôt que les coûts de production de la qualité phytosanitaire, qu'il nous semble expliquer la persistance d'une pratique de gestion du risque plus onéreuse.

Vu la hauteur de ces coûts d'affranchissement de la dépendance de sentier, certains pays exportateurs comme la France continuent à subir les coûts de maladaptation occasionnés par la nécessité de suivre une norme de facto dominante. 


\section{Références Bibliographiques}

Anderson J. E., Marcouiller D. (2002). Insecurity and the pattern of trade: An empirical investigation. Review of Economics and statistics, vol. 84, n2, pp. 342-352.

Arthur W. B. (1983). On competing technologies and historical small events: the dynamics of choice under increasing returns. IIASA WP-83-090, IIASA Laxenburg, Austria, 21 pages.

Berkowitz D., Moenius J., Pistor K. (2004). Legal institutions and international trade flows. Michigan Journal of International Law, vol. 26, pp. 163-199.

Bonizzoni M., Guglielmino C. R., Smallridge C. J., Gomulski M., Malacrida A. R., \& Gasperi G. (2004). On the origins of medfly invasion and expansion in Australia. Molecular Ecology, 13(12), pp. 3845-3855.

Casella A. (1996). On market integration and the development of institutions: the case of international commercial arbitration. European Economic Review, vol. 40, n ${ }^{\circ}$, pp. 155-186.

Castonguay S. (2005). Biorégionalisme, commerce agricole et propagation des insectes nuisibles et des maladies végétales: les conventions internationales phytopathologiques, 1878-1929. Ruralia. Sciences sociales et mondes ruraux contemporains, $\mathrm{n}^{\circ} 16 / 17,18$ pages.

Coase R. H. (1937). The nature of the firm. Economica, vol. 4, n 16 , pp. 386-405.

Coase R. H. (1960). The problem of social cost. Classic papers in natural resource economics, Palgrave Macmillan, London. pp. 87-137.

David P. A. (1985). Clio and the Economics of QWERTY. The American economic review, vol. $75, \mathrm{n}^{\circ} 2$, pp. 332-337.

De Lima C. P. F., Jessup A. J., Cruickshank L., Walsh C. J., Mansfield E. R. (2007). Cold disinfestation of citrus (Citrus spp.) for Mediterranean fruit fly (Ceratitis Capitata) and Queensland fruit fly (Bactrocera tryoni) (Diptera: Tephritidae). New Zealand Journal of Crop and Horticultural Science, vol. 35, ${ }^{\circ} 1$, pp. 39-50.

Disdier A. C., Mayer T. (2007). Je t'aime, moi non plus : Bilateral opinions and international trade. European Journal of Political Economy, vol. 23, n 4, pp. 1140-1159.

Follett P. A., Hennessey M. K. (2007). Confidence limits and sample size for determining nonhost status of fruits and vegetables to tephritid fruit flies as a quarantine measure. Journal of economic entomology, vol. 100, $\mathrm{n}^{\circ} 2$, pp. 251-257.

Foulquier E. (2007). Le froid, les agrumes et la mer. Logiques de flux, logiques d'espace dans le bassin de la Plata. Géographie, économie, société, vol. 9, n³, pp. 315-327

Granovetter M. (1985). Economic action and social structure: The problem of embeddedness. American journal of sociology, vol. 91, $\mathrm{n}^{\circ} 3$, pp. 481-510.

Jessup A. J., De Lima C. P. F., Hood C. W., Sloggett R. F., Harris A. M., Beckingham M. (1993). Quarantine disinfestation of lemons against Bactrocera tryoni and Ceratitis capitata (Diptera: Tephritidae) using cold storage. Journal of economic entomology, vol. 86, $\mathrm{n}^{\circ} 3$, pp. 798-802.

Keohane R. O. (1982). The demand for international regimes. International organization, vol. 36, $\mathrm{n}^{\circ} 2$, pp. 325-355.

Krugman P. (1994). Peddling Prosperity: Economic Sense and Nonsense in the Age of Diminished Expectations. WW Norton \& Company, 320 pages

Lamanthe A. (2007). Extension des marchés et normalisation : les systèmes agro-alimentaires dans la mondialisation. Géographie, économie, société, vol. 9, n³, pp. 257-270.

Lavallée E. (2006). Similarité institutionnelle, qualité des institutions et commerce international. Economie internationale, n ${ }^{\circ}$, pp. 27-58.

Le Velly R. (2012). Sociologie du marché. La Découverte, coll. « Repères », 125 p.

MBTOC (1995). 1994 Report of the Methyl Bromide Technical Options Committee: 1995 Assessment. UNEP, Nairobi, $304 \mathrm{p}$.

MBTOC (2011). Report of the Methyl Bromide Technical Options Committee: 2010 
Assessment of Alternatives to Methyl Bromide. UNEP, Nairobi, 387 p.

North D. C. (1990). Institutions, Institutional Change and Economic Performance. Cambridge University Press.

North D. C. (2005). Institutions and the process of economic change. Management International, vol. $9, \mathrm{n}^{\circ} 3$, pp. 1-7.

North D. C., Thomas R. P. (1973). The rise of the western world: A new economic history. Cambridge University Press.

OEPP/EPPO (1990). Exigences Spécifiques de Quarantaine. Document technique de l'OEPP $\mathrm{n}^{\circ} 1008$.

Pierson P. (2000). Increasing returns, path dependence, and the study of politics. American political science review, vol. 94, $\mathrm{n}^{\circ} 2$, pp. 251-267.

Pomeranz K., Topik S. (1999). The World that Trade Created: Society. Culture, and the World Economy, n¹400, pp. 9-11.

Rodrik D., Subramanian A., Trebbi F. (2004). Institutions rule: the primacy of institutions over geography and integration in economic development. Journal of economic growth, vol. 9, n², pp. 131-165.

Turrini A., Van Ypersele T. (2002). Traders, courts and the home bias puzzle. CEPR Discussion Paper n. 3228, CEPR, London, 29 pages.

USDA-APHIS. (2002). Cold treatment on Fruits. Federal Register, vol. 67, n¹99, pp. 6352963536.

Wilcox E. V., Hunn C. J. (1914). Cold storage for tropical fruits. Press Bulletin nº47, Hawaii Agricultural Experiment Station, University of Hawaii, 12 pages.

Williamson O. E. (1975). Markets and hierarchies. Free Press, New York.

Williamson O.E. (1985). The Economic Institutions of Capitalism. Free Press, New York. 